International Journal of Pure and Applied Mathematics

Volume 84 No. 3 2013, 251-268

ISSN: 1311-8080 (printed version); ISSN: 1314-3395 (on-line version)

url: http://www.ijpam.eu

doi: http://dx.doi.org/10.12732/ijpam.v84i3.12

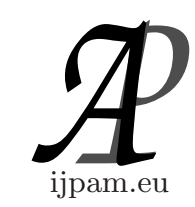

\title{
WEAK AND STRONG CONVERGENCE OF FIXED POINTS OF DEMICONTRACTIVE MAPPINGS IN SMOOTH BANACH SPACES
}

\author{
Shin Min Kang ${ }^{1}$, Arif Rafiq ${ }^{2}$, Nawab Hussain ${ }^{3}$ \\ ${ }^{1}$ Department of Mathematics and RINS \\ Gyeongsang National University \\ Jinju, 660-701, KOREA \\ ${ }^{2}$ School of CS and Mathematics \\ Hajvery University \\ 43-52 Industrial Area, Gulberg-III \\ Lahore, 54660, PAKISTAN \\ ${ }^{3}$ Department of Mathematics \\ King Abdulaziz University \\ P.O. Box 80203, Jeddah 21589, SAUDI ARABIA
}

\begin{abstract}
In this paper, it is proved that the three-step iteration process with error terms in the sense of $\mathrm{Xu}$ associated with demicontractive mappings converge weakly to a fixed point of $T$. Also, if $K$ is compact, then the convergence is strong.
\end{abstract}

AMS Subject Classification: 47H10, 47J25, 54H25

Key Words: $q$-uniformly smooth Banach spaces, demicontractive mappings, three-step iteration process

\section{Introduction and Preliminaries}

The symbols $D(T)$ and $F(T)$ stand for the domain and the set of fixed points of $T$ (for a single-valued mapping $T: X \rightarrow X, x \in X$ is called a fixed point of

Received: February 8, 2013

(c) 2013 Academic Publications, Ltd.

${ }^{\S}$ Correspondence author url: www.acadpubl.eu 
$T$ if $T x=x)$.

Let $T: D(T) \subset H \rightarrow H$ be a a mapping, where $H$ is a Hilbert space.

Definition 1.1. (see [3], [8]) (1) A mapping $T$ is said to be strictly pseudocontractive if there exists a constant $k \in[0,1)$ such that, for all $x, y \in D(T)$,

$$
\|T x-T y\|^{2} \leq\|x-y\|^{2}+k\|x-y-(T x-T y)\|^{2} .
$$

(2) If $F(T) \neq \emptyset$ and there exists a constant $k \in[0,1)$ such that, for all $x \in D(T)$ and $x^{*} \in F(T)$,

$$
\left\|T x-x^{*}\right\|^{2} \leq\left\|x-x^{*}\right\|^{2}+k\|x-T x\|^{2},
$$

then $T$ is said to be demicontractive.

It is easy to see that a strictly pseudocontractive mapping with a fixed point is demicontractive. Thus the class of demicontractive mappings properly includes the class of strictly pseudocontractive mappings with fixed points. The important class of quasi-nonexpansive mappings (where a mapping $T$ is said to be quasi-nonexpansive if $F(T) \neq \emptyset$ and

$$
\left\|T x-x^{*}\right\| \leq\left\|x-x^{*}\right\|
$$

for all $x \in D(T)$ and $\left.x^{*} \in F(T)\right)$ is also a subclass of this class of demicontractive mappings.

Remark 1.2. If we set $\lambda=\frac{(1-k)}{2}$, then it is routine to see that, in Hilbert spaces, (1.1) is equivalent to the condition: there exists a constant $\lambda>0$ such that, for all $x \in D(T)$ and $x^{*} \in F(T)$,

$$
\left\langle x-T x, x-x^{*}\right\rangle \geq \lambda\|x-T x\|^{2},
$$

which is the condition introduced by Maruster [15]. Thus the class of nonlinear mappings introduced in 1977 by Hicks and Kubicek [8] and Maruster [15] independently coincide in Hilbert spaces. It is easy to observe from (1.1) that

$$
\begin{aligned}
&\left\|T x-x^{*}\right\| \leq\left\|x-x^{*}\right\|+\sqrt{k}\|x-T x\| \\
& \leq(1+\sqrt{k})\left\|x-x^{*}\right\|+\sqrt{k}\left\|T x-x^{*}\right\|, \\
&(1-\sqrt{k})\left\|T x-x^{*}\right\| \leq(1+\sqrt{k})\left\|x-x^{*}\right\|, \\
&\left\|T x-x^{*}\right\| \leq\left(\frac{1+\sqrt{k}}{1-\sqrt{k}}\right)\left\|x-x^{*}\right\|=L\left\|x-x^{*}\right\|,
\end{aligned}
$$


where

$$
L=\frac{1+\sqrt{k}}{1-\sqrt{k}}
$$

and from (1.2) that

$$
\left\|x-x^{*}\right\| \geq \lambda\|x-T x\| \geq \lambda\left(\left\|T x-x^{*}\right\|-\left\|x-x^{*}\right\|\right)
$$

and so

$$
\left\|T x-x^{*}\right\| \leq L\left\|x-x^{*}\right\|,
$$

where $L=1+\lambda^{-1}$. Several authors have studied this class of nonlinear mappings (see, for example, ([1-24,26-27]) and convergence theorems have been established for the iteration processes of the Mann-type (see, for example, [14]).

Let $K$ be a nonempty subset of an arbitrary Banach space $X$ and $X^{*}$ be its dual space.

Definition 1.3. The modulus of smoothness of $X$ is the function $\rho_{X}$ : $[0, \infty) \rightarrow[0, \infty)$ defined by

$$
\rho_{X}(\tau):=\frac{1}{2} \sup \{\|x+y\|+\|x-y\|-2:\|x\| \leq 1,\|y\| \leq \tau\} .
$$

For any $q>1, X$ is called $q$-uniformly smooth if there exists a constant $c>0$ such that $\rho_{X}(\tau) \leq c \tau^{q}$ and $X$ is called uniformly smooth if $\lim _{\tau \rightarrow 0} \frac{\rho_{X}(\tau)}{\tau}=0$.

Clearly, every $q$-uniformly smooth Banach space is uniformly smooth. Moreover, it is well known that Hilbert spaces are 2-uniformly smooth while

$$
L_{p}\left(\text { or } \ell_{p}\right) \text { or } W_{m}^{p} \text { is } \begin{cases}p \text {-uniformly smooth } & \text { if } 1<p \leq 2, \\ 2 \text {-uniformly smooth } & \text { if } p \geq 2 .\end{cases}
$$

Definition 1.4. Let $X^{*}$ denote the dual space of $X$ and $J_{p}: X \rightarrow 2^{X^{*}}$ denote the generalized duality mapping defined by

$$
J_{p}(x):=\left\{f^{*} \in X^{*}:\left\langle x, f^{*}\right\rangle=\|x\|^{p},\left\|f^{*}\right\|=\|x\|^{p-1}\right\},
$$

where $\langle\cdot, \cdot\rangle$ denotes the generalized duality pairing.

It is known that $J_{p}$ is single-valued (denoted by $j_{p}$ ) and Lipschitz Höldercontinuous with constant $L_{*}>0$ if $X$ is $p$-uniformly smooth. That is,

$$
\left\|j_{p}(x)-j_{p}(y)\right\| \leq L_{*}\|x-y\|^{p-1}
$$

for all $x, y \in X$. Moreover, for all $x \in X$ with $x \neq 0, J_{p}(x)=\|x\|^{p-2} J_{2}(x)$, where $J_{p}=J_{2}$ is the normalized duality mapping. 
Lemma 1.5. (see [25]) If $X$ is a real $q$-uniformly smooth Banach space, then there exists positive constant $C_{q}$ such that, for all $x, y \in X$,

$$
\|x+y\|^{q} \leq\|x\|^{q}+q\left\langle y, j_{q}(x)\right\rangle+C_{q}\|y\|^{q} .
$$

Remark 1.6. ([16]) In [4], Chidume extended the condition (1.2) to arbitrary real Banach spaces $X$. If $X$ is $q$-uniformly smooth, then the condition (1.2) becomes

$$
\begin{aligned}
\left\langle x-T x, j_{q}\left(x-x^{*}\right)\right\rangle & \geq\left\|x-x^{*}\right\|^{q-2}\left\langle x-T x, j\left(x-x^{*}\right)\right\rangle \\
& \geq\left\|x-x^{*}\right\|^{q-2} \lambda\|x-T x\|^{2} \\
& \geq \lambda^{q-1}\|x-T x\|^{q}
\end{aligned}
$$

which implies that

$$
\left\langle T x-x^{*}, j_{q}\left(x-x^{*}\right)\right\rangle \leq\left\|x-x^{*}\right\|^{q}-\lambda^{q-1}\|x-T x\|^{q} .
$$

Lemma 1.7. (see [13], [22]) Suppose that $\left\{\rho_{n}\right\}$ and $\left\{\sigma_{n}\right\}$ are two sequences of nonnegative numbers such that, for some real number $n_{0} \geq 1$,

$$
\rho_{n+1} \leq \rho_{n}+\sigma_{n}
$$

for all $n \geq n_{0}$.

(a) If $\sum_{n \geq 0} \sigma_{n}<\infty$, then $\lim _{n \rightarrow \infty} \rho_{n}$ exists;

(b) If $\sum_{n \geq 0} \sigma_{n}<\infty$ and $\left\{\rho_{n}\right\}$ has a subsequence converging to zero, then $\lim _{n \rightarrow \infty} \rho_{n}=\overline{0}$.

Definition 1.8. A mapping $T: K \rightarrow X$ is said to be demiclosed at a point $z \in X$ if the weak convergence of $\left\{x_{n}\right\}$ in $K$ to some point $p \in K$ and the strong convergence of $\left\{T x_{n}\right\}$ to $z$ implies that $T p=z$.

Definition 1.9. A mapping $T: K \rightarrow X$ is said to be demicompact at a point $z \in K$ if, for any bounded sequence $\left\{x_{n}\right\}$ in $K$ such that $(I-T) x_{n} \rightarrow z$ as $n \rightarrow \infty$, then there exist a subsequence $\left\{x_{n_{j}}\right\}$ and a point $p \in K$ such that $x_{n_{j}} \rightarrow p$ as $j \rightarrow \infty$ and $(I-T) p=z$.

Let $K$ be a nonempty closed convex subset of a real $q$-uniformly smooth Banach space and $T: K \rightarrow K$ be a demicontractive mapping with $(I-T)$ demiclosed at $0 \in K$. In this paper, it is proved that the three-step iteration process with error terms in the sense of $\mathrm{Xu}$ [26] associated with demicontractive mappings converge weakly to a fixed point of $T$. Also, if $K$ is compact, then the convergence is strong. 


\section{Main Results}

We now prove our main results.

Theorem 2.1. Let $K$ be a nonempty closed convex subset of a real $q$ uniformly smooth Banach space $X$ and $T: K \rightarrow K$ be a demicontractive mapping such that $(I-T)$ is demiclosed at $0 \in K$. Let $\left\{v_{n}\right\},\left\{u_{n}\right\}$ and $\left\{w_{n}\right\}$ be three bounded sequences in $K$. Let $\left\{a_{n}\right\},\left\{b_{n}\right\},\left\{c_{n}\right\},\left\{a_{n}^{\prime}\right\},\left\{b_{n}^{\prime}\right\},\left\{c_{n}^{\prime}\right\},\left\{a_{n}^{\prime \prime}\right\}$, $\left\{b_{n}^{\prime \prime}\right\}$ and $\left\{c_{n}^{\prime \prime}\right\}$ be the real sequences in $[0,1]$ satisfying

(i) $a_{n}+b_{n}+c_{n}=a_{n}^{\prime}+b_{n}^{\prime}+c_{n}^{\prime}=a_{n}^{\prime \prime}+b_{n}^{\prime \prime}+c_{n}^{\prime \prime}=1$ for all $n \geq 0$;

(ii) $\sum_{n \geq 0} b_{n}=\infty$ and $\lim _{n \rightarrow \infty} b_{n}=0$;

(iii) $\sum_{n \geq 0} b_{n}^{\prime s}<\infty, \sum_{n \geq 0} b_{n}^{\prime \prime s}<\infty, \sum_{n \geq 0} c_{n}^{s}<\infty, \sum_{n \geq 0} c_{n}^{\prime s}<\infty$ and $\sum_{n \geq 0} c_{n}^{\prime \prime s}<\infty$, where $s=\min \{1, q-1\}$.

Then the sequence $\left\{x_{n}\right\}$ generated from an arbitrary $x_{0} \in K$ by

$$
\left\{\begin{array}{l}
x_{n+1}=a_{n} x_{n}+b_{n} T y_{n}+c_{n} v_{n} \\
y_{n}=a_{n}^{\prime} x_{n}+b_{n}^{\prime} T z_{n}+c_{n}^{\prime} u_{n} \\
z_{n}=a_{n}^{\prime \prime} x_{n}+b_{n}^{\prime \prime} T x_{n}+c_{n}^{\prime \prime} w_{n}
\end{array}\right.
$$

for all $n \geq 0$ converges weakly to a fixed point of $T$.

Proof. Let $x^{*} \in K$ be a fixed point of $T$. Then, using (1.4), we have the following estimates:

$$
\begin{aligned}
& \left\|b_{n}^{\prime \prime}\left(T x_{n}-x^{*}\right)+c_{n}^{\prime \prime}\left(w_{n}-x^{*}\right)\right\|^{q} \\
& \leq b_{n}^{\prime \prime q}\left\|T x_{n}-x^{*}\right\|^{q}+C_{q} c_{n}^{\prime \prime q}\left\|w_{n}-x^{*}\right\|^{q} \\
& \quad+q b_{n}^{\prime \prime q-1} c_{n}^{\prime \prime}\left\|w_{n}-x^{*}\right\|\left\|T x_{n}-x^{*}\right\|^{q-1} \\
& \leq L^{q} b_{n}^{\prime \prime q}\left\|x_{n}-x^{*}\right\|^{q}+C_{q} c_{n}^{\prime \prime}\left\|w_{n}-x^{*}\right\|^{q} \\
& \quad+q L^{q-1} b_{n}^{\prime \prime q-1} c_{n}^{\prime \prime}\left\|w_{n}-x^{*}\right\|\left\|x_{n}-x^{*}\right\|^{q-1}
\end{aligned}
$$

and

$$
\begin{aligned}
& \left\|j_{q}\left(x_{n}-x^{*}\right)-j_{q}\left(z_{n}-x^{*}\right)\right\| \\
& \leq L_{*}\left\|x_{n}-z_{n}\right\|^{q-1} \\
& \leq L_{*}\left[\left[(1+L) b_{n}^{\prime \prime}+c_{n}^{\prime \prime}\right]\left\|x_{n}-x^{*}\right\|+c_{n}^{\prime \prime}\left\|w_{n}-x^{*}\right\|\right]^{q-1} .
\end{aligned}
$$


Using (1.3) and (1.4), we have

$$
\begin{aligned}
&\left\|z_{n}-x^{*}\right\|^{q} \\
& \leq a_{n}^{\prime \prime q}\left\|x_{n}-x^{*}\right\|^{q}+q a_{n}^{\prime \prime q-1} b_{n}^{\prime \prime}\left\langle T x_{n}-x^{*}, j_{q}\left(x_{n}-x^{*}\right)\right\rangle \\
&+q a_{n}^{\prime \prime q-1} c_{n}^{\prime \prime}\left\langle w_{n}-x^{*}, j_{q}\left(x_{n}-x^{*}\right)\right\rangle \\
&+C_{q}\left\|b_{n}^{\prime \prime}\left(T x_{n}-x^{*}\right)+c_{n}^{\prime \prime}\left(w_{n}-x^{*}\right)\right\|^{q} \\
& \leq a_{n}^{\prime \prime q}\left\|x_{n}-x^{*}\right\|^{q}+q a_{n}^{\prime \prime q-1} b_{n}^{\prime \prime}\left(\left\|x_{n}-x^{*}\right\|^{q}-\lambda^{q-1}\left\|x_{n}-T x_{n}\right\|^{q}\right) \\
&+q a_{n}^{\prime \prime q-1} c_{n}^{\prime \prime}\left\|w_{n}-x^{*}\right\|\left\|x_{n}-x^{*}\right\|^{q-1}+C_{q}\left[L^{q} b_{n}^{\prime \prime q}\left\|x_{n}-x^{*}\right\|^{q}\right. \\
&\left.+C_{q} c_{n}^{\prime \prime q}\left\|w_{n}-x^{*}\right\|^{q}+q L^{q-1} b_{n}^{\prime \prime q-1} c_{n}^{\prime \prime}\left\|w_{n}-x^{*}\right\|\left\|x_{n}-x^{*}\right\|^{q-1}\right] \\
&=\left(a_{n}^{\prime \prime q}+q a_{n}^{\prime \prime q-1} b_{n}^{\prime \prime}+C_{q} L^{q} b_{n}^{\prime \prime q}\right)\left\|x_{n}-x^{*}\right\|^{q} \\
&-q \lambda^{q-1} a_{n}^{\prime \prime q-1} b_{n}^{\prime \prime}\left\|x_{n}-T x_{n}\right\|^{q} \\
&+q c_{n}^{\prime \prime}\left(a_{n}^{\prime \prime q-1}+C_{q} L^{q-1} b_{n}^{\prime \prime q-1}\right)\left\|w_{n}-x^{*}\right\|\left\|x_{n}-x^{*}\right\|^{q-1} \\
&+C_{q}^{2} c_{n}^{\prime \prime q}\left\|w_{n}-x^{*}\right\|^{q} \\
& \leq\left\|x_{n}-x^{*}\right\|^{q}-q \lambda^{q-1} a_{n}^{\prime \prime q-1} b_{n}^{\prime \prime}\left\|x_{n}-T x_{n}\right\|^{q}+C_{q}^{2} c_{n}^{\prime \prime}\left\|w_{n}-x^{*}\right\|^{q} \\
&+q c_{n}^{\prime \prime}\left(a_{n}^{\prime \prime q-1}+C_{q} L^{q-1} b_{n}^{\prime \prime q-1}\right)\left\|w_{n}-x^{*}\right\|\left\|x_{n}-x^{*}\right\|^{q-1} .
\end{aligned}
$$

The last inequality [16] follows from the fact that, for all $x, y \in[0,1]$,

$$
\begin{aligned}
f(x, y) & =(1-x-y)^{q}+q(1-x-y)^{q-1} x+C_{q} L^{q} x^{q} \\
& \leq(1-x)^{q}+q(1-x)^{q-1} x+C_{q} L^{q} x^{q} \\
& \leq 1 .
\end{aligned}
$$

Indeed, $g(x)=(1-x)^{q}+q(1-x)^{q-1} x+C_{q} L^{q} x^{q}$ is monotone increasing on $[0,1]$. Therefore, $g(x) \leq g(1)=C_{q} L_{q}$. Unfortunately, $C_{q} L_{q}$ need not be less than or equal to 1 . However, since $\sum_{n>0} b_{n}^{\prime \prime}<\infty, \lim _{n \rightarrow \infty} b_{n}^{\prime \prime}=0$ and so, for all $n$ sufficiently large, $g\left(b_{n}^{\prime \prime}\right)<1$. Observe that

$$
\begin{aligned}
\left\|z_{n}-x^{*}\right\| \leq & \left\|z_{n}-x_{n}\right\|+\left\|x_{n}-x^{*}\right\| \\
\leq & {\left[(1+L) b_{n}^{\prime \prime}+c_{n}^{\prime \prime}+1\right]\left\|x_{n}-x^{*}\right\|+c_{n}^{\prime \prime}\left\|w_{n}-x^{*}\right\|, } \\
\| & b_{n}^{\prime}\left(T z_{n}-x^{*}\right)+c_{n}^{\prime}\left(u_{n}-x^{*}\right) \|^{q} \\
\leq & b_{n}^{\prime q}\left\|T z_{n}-x^{*}\right\|^{q}+C_{q} c_{n}^{q}\left\|u_{n}-x^{*}\right\|^{q} \\
& +q b_{n}^{\prime q-1} c_{n}^{\prime}\left\|u_{n}-x^{*}\right\|\left\|T z_{n}-x^{*}\right\|^{q-1} \\
\leq & L^{q} b_{n}^{\prime q}\left\|z_{n}-x^{*}\right\|^{q}+C_{q} c_{n}^{q}\left\|u_{n}-x^{*}\right\|^{q} \\
& +q L^{q-1} b_{n}^{\prime q-1} c_{n}^{\prime}\left\|u_{n}-x^{*}\right\|\left\|z_{n}-x^{*}\right\|^{q-1}
\end{aligned}
$$


and

$$
\begin{aligned}
& \left\|y_{n}-x^{*}\right\|^{q} \leq a_{n}^{\prime q}\left\|x_{n}-x^{*}\right\|^{q}+C_{q}\left\|b_{n}^{\prime}\left(T z_{n}-x^{*}\right)+c_{n}^{\prime}\left(u_{n}-x^{*}\right)\right\|^{q} \\
& +q a_{n}^{\prime q-1} b_{n}^{\prime}\left\langle T z_{n}-x^{*}, j_{q}\left(x_{n}-x^{*}\right)\right\rangle+q a_{n}^{\prime q-1} c_{n}^{\prime}\left\langle u_{n}-x^{*}, j_{q}\left(x_{n}-x^{*}\right)\right\rangle \\
& \leq a_{n}^{\prime q}\left\|x_{n}-x^{*}\right\|^{q}+C_{q}\left[L^{q} b_{n}^{\prime q}\left\|z_{n}-x^{*}\right\|^{q}+C_{q} c_{n}^{\prime q}\left\|u_{n}-x^{*}\right\|^{q}\right. \\
& \left.+q L^{q-1} b_{n}^{\prime q-1} c_{n}^{\prime}\left\|u_{n}-x^{*}\right\|\left\|z_{n}-x^{*}\right\|^{q-1}\right] \\
& +q a_{n}^{\prime q-1} b_{n}^{\prime}\left\langle T z_{n}-x^{*}, j_{q}\left(z_{n}-x^{*}\right)\right\rangle \\
& +q a_{n}^{\prime q-1} b_{n}^{\prime}\left\langle T z_{n}-x^{*}, j_{q}\left(x_{n}-x^{*}\right)-j_{q}\left(z_{n}-x^{*}\right)\right\rangle \\
& +q a_{n}^{\prime q-1} c_{n}^{\prime}\left\|u_{n}-x^{*}\right\|\left\|x_{n}-x^{*}\right\|^{q-1} \\
& \leq a_{n}^{\prime q}\left\|x_{n}-x^{*}\right\|^{q}+q a_{n}^{\prime q-1} b_{n}^{\prime}\left(\left\|z_{n}-x^{*}\right\|^{q}-\lambda^{q-1}\left\|z_{n}-T z_{n}\right\|^{q}\right) \\
& +q a_{n}^{\prime q-1} b_{n}^{\prime}\left\|T z_{n}-x^{*}\right\|\left\|j_{q}\left(x_{n}-x^{*}\right)-j_{q}\left(z_{n}-x^{*}\right)\right\| \\
& +q a_{n}^{\prime q-1} c_{n}^{\prime}\left\|u_{n}-x^{*}\right\|\left\|x_{n}-x^{*}\right\|^{q-1}+C_{q} L^{q} b_{n}^{\prime q}\left\|z_{n}-x^{*}\right\|^{q} \\
& +C_{q}^{2} c_{n}^{\prime q}\left\|u_{n}-x^{*}\right\|^{q}+q C_{q} L^{q-1} b_{n}^{\prime q-1} c_{n}^{\prime}\left\|u_{n}-x^{*}\right\|\left\|z_{n}-x^{*}\right\|^{q-1} \\
& \leq a_{n}^{\prime q}\left\|x_{n}-x^{*}\right\|^{q}+q a_{n}^{\prime q-1} b_{n}^{\prime}\left\|z_{n}-x^{*}\right\|^{q}-q \lambda^{q-1} a_{n}^{\prime q-1} b_{n}^{\prime}\left\|z_{n}-T z_{n}\right\|^{q} \\
& +q L L_{*} a_{n}^{\prime q-1} b_{n}^{\prime}\left[\left[(1+L) b_{n}^{\prime \prime}+c_{n}^{\prime \prime}+1\right]\left\|x_{n}-x^{*}\right\|+c_{n}^{\prime \prime}\left\|w_{n}-x^{*}\right\|\right] \\
& \times\left[\left[(1+L) b_{n}^{\prime \prime}+c_{n}^{\prime \prime}\right]\left\|x_{n}-x^{*}\right\|+c_{n}^{\prime \prime}\left\|w_{n}-x^{*}\right\|\right]^{q-1} \\
& +q a_{n}^{\prime q-1} c_{n}^{\prime}\left\|u_{n}-x^{*}\right\|\left\|x_{n}-x^{*}\right\|^{q-1}+C_{q} L^{q} b_{n}^{\prime q}\left\|z_{n}-x^{*}\right\|^{q} \\
& +C_{q}^{2} c_{n}^{\prime q}\left\|u_{n}-x^{*}\right\|^{q}+q C_{q} L^{q-1} b_{n}^{\prime q-1} c_{n}^{\prime}\left\|u_{n}-x^{*}\right\|\left\|z_{n}-x^{*}\right\|^{q-1} \\
& =\left(a_{n}^{\prime q}+q a_{n}^{\prime q-1} b_{n}^{\prime}+C_{q} L^{q} b_{n}^{\prime q}\right)\left\|x_{n}-x^{*}\right\|^{q}-q \lambda^{q-1} a_{n}^{\prime q-1} b_{n}^{\prime}\left\|z_{n}-T z_{n}\right\|^{q} \\
& -q \lambda^{q-1} a_{n}^{\prime \prime q-1}\left(q a_{n}^{\prime q-1}+C_{q} L^{q} b_{n}^{\prime q-1}\right) b_{n}^{\prime} b_{n}^{\prime \prime}\left\|x_{n}-T x_{n}\right\|^{q} \\
& +q\left(a_{n}^{\prime \prime q-1}+C_{q} L^{q-1} b_{n}^{\prime \prime q-1}\right)\left(q a_{n}^{\prime q-1}+C_{q} L^{q} b_{n}^{\prime q-1}\right) b_{n}^{\prime} c_{n}^{\prime \prime}\left\|w_{n}-x^{*}\right\| \\
& \times\left\|z_{n}-x^{*}\right\|^{q-1}+C_{q}^{2}\left(q a_{n}^{\prime q-1}+C_{q} L^{q} b_{n}^{\prime q-1}\right) b_{n}^{\prime} c_{n}^{\prime \prime q}\left\|w_{n}-x^{*}\right\|^{q} \\
& +C_{q}^{2} c_{n}^{\prime q}\left\|u_{n}-x^{*}\right\|^{q}+q L L_{*} a_{n}^{\prime q-1} b_{n}^{\prime}\left[\left[(1+L) b_{n}^{\prime \prime}+c_{n}^{\prime \prime}+1\right]\left\|x_{n}-x^{*}\right\|\right. \\
& \left.+c_{n}^{\prime \prime}\left\|w_{n}-x^{*}\right\|\right]\left[\left[(1+L) b_{n}^{\prime \prime}+c_{n}^{\prime \prime}\right]\left\|x_{n}-x^{*}\right\|+c_{n}^{\prime \prime}\left\|w_{n}-x^{*}\right\|\right]^{q-1} \\
& +q C_{q} L^{q-1} b_{n}^{\prime q-1} c_{n}^{\prime}\left\|u_{n}-x^{*}\right\|\left[\left[(1+L) b_{n}^{\prime \prime}+c_{n}^{\prime \prime}+1\right]\left\|x_{n}-x^{*}\right\|\right. \\
& \left.+c_{n}^{\prime \prime}\left\|w_{n}-x^{*}\right\|\right]^{q-1}+q a_{n}^{\prime q-1} c_{n}^{\prime}\left\|u_{n}-x^{*}\right\|\left\|x_{n}-x^{*}\right\|^{q-1} \\
& \leq\left\|x_{n}-x^{*}\right\|^{q}-q \lambda^{q-1} a_{n}^{\prime q-1} b_{n}^{\prime}\left\|z_{n}-T z_{n}\right\|^{q} \\
& -q \lambda^{q-1} a_{n}^{\prime \prime q-1}\left(q a_{n}^{\prime q-1}+C_{q} L^{q} b_{n}^{\prime q-1}\right) b_{n}^{\prime} b_{n}^{\prime \prime}\left\|x_{n}-T x_{n}\right\|^{q} \\
& +q\left(a_{n}^{\prime \prime q-1}+C_{q} L^{q-1} b_{n}^{\prime \prime q-1}\right)\left(q a_{n}^{\prime q-1}+C_{q} L^{q} b_{n}^{\prime q-1}\right) b_{n}^{\prime} c_{n}^{\prime \prime}\left\|w_{n}-x^{*}\right\| \\
& \times\left\|z_{n}-x^{*}\right\|^{q-1}+C_{q}^{2}\left(q a_{n}^{\prime q-1}+C_{q} L^{q} b_{n}^{\prime q-1}\right) b_{n}^{\prime} c_{n}^{\prime \prime q}\left\|w_{n}-x^{*}\right\|^{q} \\
& +C_{q}^{2} c_{n}^{\prime q}\left\|u_{n}-x^{*}\right\|^{q}+q L L_{*} a_{n}^{\prime q-1} b_{n}^{\prime}\left[\left[(1+L) b_{n}^{\prime \prime}+c_{n}^{\prime \prime}+1\right]\left\|x_{n}-x^{*}\right\|\right.
\end{aligned}
$$




$$
\begin{aligned}
& \left.+c_{n}^{\prime \prime}\left\|w_{n}-x^{*}\right\|\right]\left[\left[(1+L) b_{n}^{\prime \prime}+c_{n}^{\prime \prime}\right]\left\|x_{n}-x^{*}\right\|+c_{n}^{\prime \prime}\left\|w_{n}-x^{*}\right\|\right]^{q-1} \\
& +q C_{q} L^{q-1} b_{n}^{\prime q-1} c_{n}^{\prime}\left\|u_{n}-x^{*}\right\|\left[\left[(1+L) b_{n}^{\prime \prime}+c_{n}^{\prime \prime}+1\right]\left\|x_{n}-x^{*}\right\|\right. \\
& \left.+c_{n}^{\prime \prime}\left\|w_{n}-x^{*}\right\|\right]^{q-1}+q a_{n}^{\prime q-1} c_{n}^{\prime}\left\|u_{n}-x^{*}\right\|\left\|x_{n}-x^{*}\right\|^{q-1}
\end{aligned}
$$

Now, also we have

$$
\begin{aligned}
\left\|b_{n}\left(T y_{n}-x^{*}\right)+c_{n}\left(v_{n}-x^{*}\right)\right\|^{q} \leq & b_{n}^{q}\left\|T y_{n}-x^{*}\right\|^{q}+C_{q} c_{n}^{q}\left\|v_{n}-x^{*}\right\|^{q} \\
& +q b_{n}^{q-1} c_{n}\left\|v_{n}-x^{*}\right\|\left\|T y_{n}-x^{*}\right\|^{q-1} \\
\leq & L_{q} b_{n}^{q}\left\|y_{n}-x^{*}\right\|^{q}+C_{q} c_{n}^{q}\left\|v_{n}-x^{*}\right\|^{q} \\
& +q L^{q-1} b_{n}^{q-1} c_{n}\left\|v_{n}-x^{*}\right\|\left\|y_{n}-x^{*}\right\|^{q-1}
\end{aligned}
$$

$$
\begin{aligned}
\left\|j_{q}\left(x_{n}-x^{*}\right)-j_{q}\left(y_{n}-x^{*}\right)\right\| \leq & L_{*}\left\|x_{n}-y_{n}\right\|^{q-1} \\
\leq & L_{*}\left[\left[\left[1+L\left[(1+L) b_{n}^{\prime \prime}+c_{n}^{\prime \prime}+1\right]\right] b_{n}^{\prime}+c_{n}^{\prime}\right]\left\|x_{n}-x^{*}\right\|\right. \\
& \left.+L b_{n}^{\prime} c_{n}^{\prime \prime}\left\|w_{n}-x^{*}\right\|+c_{n}^{\prime}\left\|u_{n}-x^{*}\right\|\right]^{q-1}, \\
\left\|y_{n}-x^{*}\right\| \leq & \left\|y_{n}-x_{n}\right\|+\left\|x_{n}-x^{*}\right\| \\
\leq & {\left[\left[1+L\left[(1+L) b_{n}^{\prime \prime}+c_{n}^{\prime \prime}+1\right]\right] b_{n}^{\prime}+c_{n}^{\prime}+1\right]\left\|x_{n}-x^{*}\right\| } \\
& +L b_{n}^{\prime} c_{n}^{\prime \prime}\left\|w_{n}-x^{*}\right\|+c_{n}^{\prime}\left\|u_{n}-x^{*}\right\|,
\end{aligned}
$$

$$
\begin{aligned}
&\left\|x_{n+1}-x^{*}\right\|^{q} \\
& \leq a_{n}^{q}\left\|x_{n}-x^{*}\right\|^{q}+C_{q}\left\|b_{n}\left(T y_{n}-x^{*}\right)+c_{n}\left(v_{n}-x^{*}\right)\right\|^{q} \\
&+q a_{n}^{q-1} b_{n}\left\langle T y_{n}-x^{*}, j_{q}\left(x_{n}-x^{*}\right)\right\rangle+q a_{n}^{q-1} c_{n}\left\langle v_{n}-x^{*}, j_{q}\left(x_{n}-x^{*}\right)\right\rangle \\
& \leq a_{n}^{q}\left\|x_{n}-x^{*}\right\|^{q}+C_{q}\left[b_{n}^{q}\left\|T y_{n}-x^{*}\right\|^{q}+C_{q} c_{n}^{q}\left\|v_{n}-x^{*}\right\|^{q}\right. \\
&\left.+q b_{n}^{q-1} c_{n}\left\|v_{n}-x^{*}\right\|\left\|T y_{n}-x^{*}\right\|^{q-1}\right] \\
&+q a_{n}^{q-1} b_{n}\left\langle T y_{n}-x^{*}, j_{q}\left(y_{n}-x^{*}\right)\right\rangle \\
&+q a_{n}^{q-1} b_{n}\left\langle T y_{n}-x^{*}, j_{q}\left(x_{n}-x^{*}\right)-j_{q}\left(y_{n}-x^{*}\right)\right\rangle \\
&+q a_{n}^{q-1} c_{n}\left\|v_{n}-x^{*}\right\|\left\|x_{n}-x^{*}\right\|^{q-1} \\
& \leq a_{n}^{q}\left\|x_{n}-x^{*}\right\|^{q}+q a_{n}^{q-1} b_{n}\left(\left\|y_{n}-x^{*}\right\|^{q}-\lambda^{q-1}\left\|y_{n}-T y_{n}\right\|^{q}\right) \\
&+q L a_{n}^{q-1} b_{n}\left\|y_{n}-x^{*}\right\|\left\|j_{q}\left(x_{n}-x^{*}\right)-j_{q}\left(y_{n}-x^{*}\right)\right\| \\
&+q a_{n}^{q-1} c_{n}\left\|v_{n}-x^{*}\right\|\left\|x_{n}-x^{*}\right\|^{q-1}+C_{q} L^{q} b_{n}^{q}\left\|y_{n}-x^{*}\right\|^{q} \\
&+C_{q}^{2} c_{n}^{q}\left\|v_{n}-x^{*}\right\|^{q}+q C_{q} L^{q-1} b_{n}^{q-1} c_{n}\left\|v_{n}-x^{*}\right\|\left\|y_{n}-x^{*}\right\|^{q-1} \\
&
\end{aligned}
$$




$$
\begin{aligned}
\leq & \left(a_{n}^{q}+q a_{n}^{q-1} b_{n}+C_{q} L^{q} b_{n}^{q}\right)\left\|x_{n}-x^{*}\right\|^{q} \\
- & q \lambda^{q-1} a_{n}^{\prime q-1}\left(q a_{n}^{q-1}+C_{q} L^{q} b_{n}^{q-1}\right) b_{n} b_{n}^{\prime}\left\|z_{n}-T z_{n}\right\|^{q} \\
- & q \lambda^{q-1} a_{n}^{\prime \prime}{ }^{q-1}\left(q a_{n}^{\prime q-1}+C_{q} L^{q} b_{n}^{q-1}\right)\left(q a_{n}^{q-1}+C_{q} L^{q} b_{n}^{q-1}\right) \\
& \times b_{n} b_{n}^{\prime} b_{n}^{\prime \prime}\left\|x_{n}-T x_{n}\right\|^{q}-q \lambda^{q-1} a_{n}^{q-1} b_{n}\left\|y_{n}-T y_{n}\right\|^{q} \\
+ & q\left(a_{n}^{\prime \prime}{ }^{q-1}+C_{q} L^{q-1} b_{n}^{\prime \prime q-1}\right)\left(q a_{n}^{q-1}+C_{q} L^{q} b_{n}^{\prime q-1}\right) \\
& \times\left(q a_{n}^{q-1}+C_{q} L^{q} b_{n}^{q-1}\right) b_{n} b_{n}^{\prime} c_{n}^{\prime \prime}\left\|w_{n}-x^{*}\right\|\left\|x_{n}-x^{*}\right\|^{q-1} \\
+ & C_{q}^{2}\left(q a_{n}^{\prime q-1}+C_{q} L^{q} b_{n}^{q-1}\right)\left(q a_{n}^{q-1}+C_{q} L^{q} b_{n}^{q-1}\right) b_{n} b_{n}^{\prime} c_{n}^{\prime \prime}\left\|w_{n}-x^{*}\right\|^{q} \\
+ & C_{q}^{2}\left(q a_{n}^{q-1}+C_{q} L^{q} b_{n}^{q-1}\right) b_{n} c_{n}^{q}\left\|u_{n}-x^{*}\right\|^{q} \\
+ & q\left(q a_{n}^{q-1}+C_{q} L^{q} b_{n}^{q-1}\right) a_{n}^{q-1} b_{n} c_{n}^{\prime}\left\|u_{n}-x^{*}\right\|\left\|x_{n}-x^{*}\right\|^{q-1} \\
+ & q a_{n}^{q-1} c_{n}\left\|v_{n}-x^{*}\right\| \cdot\left\|x_{n}-x^{*}\right\|^{q-1}+C_{q}^{2} c_{n}^{q}\left\|v_{n}-x^{*}\right\|^{q} \\
+ & q L L_{*}\left(q a_{n}^{q-1}+C_{q} L^{q} b_{n}^{q-1}\right) a_{n}^{\prime q-1} b_{n} b_{n}^{\prime}\left(\left[(L+1) b_{n}^{\prime \prime}+c_{n}^{\prime \prime}\right]\left\|x_{n}-x^{*}\right\|\right. \\
+ & \left.c_{n}^{\prime \prime}\left\|w_{n}-x^{*}\right\|\right)^{q-1}\left(\left[(L+1) b_{n}^{\prime \prime}+c_{n}^{\prime \prime}+1\right]\left\|x_{n}-x^{*}\right\|+c_{n}^{\prime \prime}\left\|w_{n}-x^{*}\right\|\right) \\
+ & q C_{q} L^{q-1}\left(q a_{n}^{q-1}+C_{q} L^{q} b_{n}^{q-1}\right) b_{n}^{\prime q-1} b_{n} c_{n}^{\prime}\left\|u_{n}-x^{*}\right\| \\
& \times\left(\left[(L+1) b_{n}^{\prime \prime}+c_{n}^{\prime \prime}+1\right]\left\|x_{n}-x^{*}\right\|+c_{n}^{\prime \prime}\left\|w_{n}-x^{*}\right\|\right)^{q-1} \\
+ & q L L_{*} a_{n}^{q-1} b_{n}\left(\left[1+L\left[(L+1) b_{n}^{\prime \prime}+c_{n}^{\prime \prime}+1\right] b_{n}^{\prime}+c_{n}^{\prime}+1\right]\left\|x_{n}-x^{*}\right\|\right. \\
+ & \left.L b_{n}^{\prime} c_{n}^{\prime \prime}\left\|w_{n}-x^{*}\right\|+c_{n}^{\prime}\left\|u_{n}-x^{*}\right\|\right) \\
& \times\left(\left[1+L\left[(L+1) b_{n}^{\prime \prime}+c_{n}^{\prime \prime}+1\right] b_{n}^{\prime}+c_{n}^{\prime}\right]\left\|x_{n}-x^{*}\right\|+L b_{n}^{\prime} c_{n}^{\prime \prime}\left\|w_{n}-x^{*}\right\|\right. \\
+ & \left.c_{n}^{\prime}\left\|u_{n}-x^{*}\right\|\right)^{q-1}+q C_{q} L^{q-1} b_{n}^{q-1} c_{n}\left\|v_{n}-x^{*}\right\| \\
& \times\left(\left[1+L\left[(L+1) b_{n}^{\prime \prime}+c_{n}^{\prime \prime}+1\right] b_{n}^{\prime}+c_{n}^{\prime}+1\right]\left\|x_{n}-x^{*}\right\|\right. \\
+ & \left.L b_{n}^{\prime} c_{n}^{\prime \prime}\left\|w_{n}-x^{*}\right\|+c_{n}^{\prime}\left\|u_{n}-x^{*}\right\|\right)^{q-1} \cdot \\
& \times(L)
\end{aligned}
$$

Let

$$
M=\max \left\{\sup _{n \geq 0}\left\{\left\|u_{n}-x^{*}\right\|\right\}, \sup _{n \geq 0}\left\{\left\|v_{n}-x^{*}\right\|\right\}, \sup _{n \geq 0}\left\{\left\|w_{n}-x^{*}\right\|\right\}\right\} .
$$

Now, we consider the following two cases:

Case 1: For all $n \geq 0,\left\|x_{n}-x^{*}\right\| \geq M$. Then we have

$$
\left\|x_{n+1}-x^{*}\right\|^{q} \leq\left(1+\gamma_{n}\right)\left\|x_{n}-x^{*}\right\|^{q},
$$

where

$$
\gamma_{n}=f\left(b_{n}^{\prime s}, b_{n}^{\prime \prime s}, c_{n}^{s}, c_{n}^{\prime s}, c_{n}^{\prime \prime s}\right), \quad \sum_{n=1}^{\infty} \gamma_{n}<\infty .
$$

Case 2: For all $n \geq 0,\left\|x_{n}-x^{*}\right\| \leq M$. Then we know that, for a constant $M_{1} \geq M^{q}$

$$
\left\|x_{n+1}-x^{*}\right\|^{q} \leq\left\|x_{n}-x^{*}\right\|^{q}+M_{1} \gamma_{n}
$$


Thus, according to Lemma $1.7, \lim _{n \rightarrow \infty}\left\|x_{n}-x^{*}\right\|$ exists and so the sequences $\left\{x_{n}\right\},\left\{y_{n}\right\},\left\{z_{n}\right\},\left\{T x_{n}\right\},\left\{T y_{n}\right\}$ and $\left\{T z_{n}\right\}$ are bounded. Furthermore, for a positive constant $M_{0}$, we have

$$
q \lambda^{q-1} a_{n}^{q-1} b_{n}\left\|y_{n}-T y_{n}\right\|^{q} \leq\left\|x_{n}-x^{*}\right\|^{q}-\left\|x_{n+1}-x^{*}\right\|^{q}+M_{0} \gamma_{n} .
$$

Thus, iterating downwards, we have

$$
q \lambda^{q-1} \sum_{n \geq 0} a_{n}^{q-1} b_{n}\left\|y_{n}-T y_{n}\right\|^{q} \leq\left\|x_{0}-x^{*}\right\|^{q}+M_{0} \sum_{n \geq 0} \gamma_{n}<\infty
$$

and so, from the hypothesis,

$$
\liminf _{n \rightarrow \infty}\left\|y_{n}-T y_{n}\right\|=0
$$

and so there exists a subsequence $\left\{y_{n_{j}}\right\} \subset\left\{y_{n}\right\}$ such that

$$
y_{n_{j}} \rightarrow p, \quad y_{n_{j}}-T y_{n_{j}} \rightarrow 0
$$

as $j \rightarrow \infty$. Since $(I-T)$ is demiclosed at $0 \in K$ and $K$ is weakly closed, it follows that $p \in F(T)$. Thus, for any $f^{*} \in X^{*}$, we have

$$
f^{*}\left(y_{n_{j}}-p\right) \rightarrow 0
$$

as $j \rightarrow \infty$. Now, we consider

$$
\begin{aligned}
f^{*}\left(x_{n_{j}}-p\right) & =f^{*}\left(x_{n_{j}}-y_{n_{j}}\right)+f^{*}\left(y_{n_{j}}-p\right) \\
& =b_{n_{j}}^{\prime} f^{*}\left(x_{n_{j}}-T z_{n_{j}}\right)+c_{n_{j}}^{\prime} f^{*}\left(x_{n_{j}}-u_{n_{j}}\right)+f^{*}\left(y_{n_{j}}-p\right)
\end{aligned}
$$

and so

$$
\begin{aligned}
\lim _{j \rightarrow \infty}\left|f^{*}\left(x_{n_{j}}-p\right)\right| \leq & \lim _{j \rightarrow \infty}\left[b_{n_{j}}^{\prime}\left|f^{*}\left(x_{n_{j}}-T z_{n_{j}}\right)\right|+c_{n_{j}}^{\prime}\left|f^{*}\left(x_{n_{j}}-u_{n_{j}}\right)\right|\right. \\
& \left.+\left|f^{*}\left(y_{n_{j}}-p\right)\right|\right] \\
= & 0 .
\end{aligned}
$$

Thus we have $x_{n_{j}} \rightarrow p$ as $j \rightarrow \infty$. Observe that, for a constant $M_{0}>0$,

$$
\begin{aligned}
\left\|x_{n}-T y_{n}\right\| & \leq\left\|x_{n}-y_{n}\right\|+\left\|y_{n}-T y_{n}\right\| \\
& \leq\left\|y_{n}-T y_{n}\right\|+b_{n}^{\prime}\left\|x_{n}-T z_{n}\right\|+c_{n}^{\prime}\left\|x_{n}-u_{n}\right\| \\
& \leq\left\|y_{n}-T y_{n}\right\|+M_{0}\left(b_{n}^{\prime}+c_{n}^{\prime}\right) .
\end{aligned}
$$


Hence we have

$$
\lim _{j \rightarrow \infty}\left\|x_{n_{j}}-T y_{n_{j}}\right\|=0 .
$$

We now claim that, for all $k \geq 0$,

$$
f^{*}\left(x_{n_{j}+k}-p\right) \rightarrow 0
$$

as $j \rightarrow \infty$. Suppose that the claim is true for some $k=m$. Then, from

$$
\begin{aligned}
f^{*}\left(y_{n_{j}+m}-p\right)= & b_{n_{j}+m}^{\prime} f^{*}\left(T z_{n_{j}+m}-x_{n_{j}+m}\right) \\
& +c_{n_{j}+m}^{\prime} f^{*}\left(u_{n_{j}+m}-x_{n_{j}+m}\right)+f^{*}\left(x_{n_{j}+m}-p\right),
\end{aligned}
$$

we see that

$$
y_{n_{j}+m} \rightarrow p
$$

as $j \rightarrow \infty$ and so, additionally, from

$$
\begin{aligned}
f^{*}\left(x_{n_{j}+m+1}-p\right)= & b_{n_{j}+m} f^{*}\left(T y_{n_{j}+m}-x_{n_{j}+m}\right) \\
& +c_{n_{j}+m} f^{*}\left(v_{n_{j}+m}-x_{n_{j}+m}\right)+f^{*}\left(x_{n_{j}+m}-p\right),
\end{aligned}
$$

it follows that

$$
\begin{aligned}
& \left|f^{*}\left(x_{n_{j}+m+1}-p\right)\right| \\
& \leq M c_{n_{j}+m}+\left|f^{*}\left(x_{n_{j}+m}-p\right)\right|+\left\|f^{*}\right\|\left\|x_{n_{j}+m}-T y_{n_{j}+m}\right\| \\
& \rightarrow 0
\end{aligned}
$$

as $j \rightarrow \infty$. Since the claim is trivially true for $k=0$, it follows from the inductive hypothesis that the claim holds for all $k>0$. Hence $\left\{x_{n}\right\}$ converges weakly to a fixed point of $T$. This completes the proof.

Theorem 2.2. Suppose that all the conditions in Theorem 2.1 hold. If $K$ is a compact subset of $X$, then the sequence $\left\{x_{n}\right\}$ converges strongly to a fixed point of $T$.

Proof. Proceeding as in the proof of Theorem 2.1, we can show that

$$
\liminf _{n \rightarrow \infty}\left\|y_{n}-T y_{n}\right\|=0
$$

This immediately implies that a subsequence $\left\{y_{n_{j}}\right\}$ of $\left\{y_{n}\right\}$ converges strongly to a fixed point of $T$, say $p$. From the fact that, for a constant $M_{0} \geq 0$,

$$
\left\|x_{n}-p\right\| \leq\left\|x_{n}-y_{n}\right\|+\left\|y_{n}-p\right\|
$$




$$
\begin{aligned}
& \leq b_{n}^{\prime}\left\|x_{n}-T z_{n}\right\|+c_{n}^{\prime}\left\|x_{n}-u_{n}\right\|+\left\|y_{n}-p\right\| \\
& \leq M_{0}\left(b_{n}^{\prime}+c_{n}^{\prime}\right)+\left\|y_{n}-p\right\|,
\end{aligned}
$$

it follows that $\left\{x_{n_{j}}\right\}$ also converges strongly to $p$. This implies that

$$
\liminf _{n \rightarrow \infty}\left\|x_{n}-p\right\|=0
$$

Observe that, from the hypotheses, $\sigma_{n}=M_{0} \gamma_{n}$ is summable and, putting $\Phi_{n}=\left\|x_{n}-p\right\|^{q}$, we have

$$
\Phi_{n+1} \leq \Phi_{n}+\sigma_{n}
$$

Thus, following the approach of [16], for any $\varepsilon>0$, there exists an integer $j_{0}$ sufficiently large such that

$$
\Phi_{n_{j}} \leq \frac{\varepsilon}{4}
$$

for all $j \geq j_{0}$ and there exists another integer $n_{1}$ sufficiently large such that

$$
\sum_{n \geq n_{1}} \sigma_{n} \leq \frac{\varepsilon}{4}
$$

since the tail of a summable series is arbitrarily small. Choose $j_{*}$ sufficiently large such that

$$
n_{j_{*}} \geq \max \left\{n_{j_{0}}, N_{1}\right\}
$$

Then, for any $k \geq 0$, we have

$$
\Phi_{n_{j_{*}}+k+1} \leq \Phi_{n_{j_{*}}}+\sum_{r=0}^{k+1} \sigma_{n_{j_{*}}+r} \leq \frac{\varepsilon}{4}+\frac{\varepsilon}{4}=\frac{\varepsilon}{2} .
$$

Since $\varepsilon>0$ is arbitrary, it follows that $x_{n} \rightarrow x^{*}$ strongly as $n \rightarrow \infty$. This completes the proof.

Corollary 2.3. Let $X, K, T,\left\{v_{n}\right\}$ and $\left\{u_{n}\right\}$ be as in Theorem 2.1. Let $\left\{a_{n}\right\},\left\{b_{n}\right\},\left\{c_{n}\right\},\left\{a_{n}^{\prime}\right\},\left\{b_{n}^{\prime}\right\}$ and $\left\{c_{n}^{\prime}\right\}$ be the real sequences in $[0,1]$ satisfying

(i) $a_{n}+b_{n}+c_{n}=a_{n}^{\prime}+b_{n}^{\prime}+c_{n}^{\prime}=1$ for all $n \geq 0$;

(ii) $\sum_{n \geq 0} b_{n}=\infty$ and $\lim _{n \rightarrow \infty} b_{n}=0$; $1\}$.

(iii) $\sum_{n \geq 0} b_{n}^{\prime s}<\infty, \sum_{n \geq 0} c_{n}<\infty$ and $\sum_{n \geq 0} c_{n}^{\prime s}<\infty$, where $s=\min \{1, q-$

Then the sequence $\left\{x_{n}\right\}$ generated from an arbitrary $x_{0} \in K$ by

$$
\left\{\begin{array}{l}
x_{n+1}=a_{n} x_{n}+b_{n} T y_{n}+c_{n} v_{n}, \\
y_{n}=a_{n}^{\prime} x_{n}+b_{n}^{\prime} T x_{n}+c_{n}^{\prime} u_{n}
\end{array}\right.
$$

for all $n \geq 0$ converges weakly to a fixed point of $T$. 
Corollary 2.4. Suppose that all the conditions in Corollary 2.3 hold. If $K$ is a compact subset of $X$, then the sequence $\left\{x_{n}\right\}$ converges strongly to a fixed point of $T$.

Corollary 2.5. Let $X, K, T$ and $\left\{v_{n}\right\}$ be as in Theorem 2.1. Let $\left\{a_{n}\right\}$, $\left\{b_{n}\right\}$ and $\left\{c_{n}\right\}$ be real sequences in $[0,1]$ satisfying

(i) $a_{n}+b_{n}+c_{n}=1$ for all $n \geq 0$;

(ii) $\sum_{n>0} b_{n}=\infty$ and $\lim _{n \rightarrow \infty} b_{n}=0$;

(iii) $\sum_{n \geq 0} c_{n}<\infty$.

Then the sequence $\left\{x_{n}\right\}$ generated from an arbitrary $x_{0} \in K$ by

$$
x_{n+1}=a_{n} x_{n}+b_{n} T x_{n}+c_{n} v_{n}
$$

for all $n \geq 0$ converges weakly to a fixed point of $T$.

Corollary 2.6. Suppose that all the conditions in Corollary 2.5 hold. If $K$ is a compact subset of $X$, then the sequence $\left\{x_{n}\right\}$ converges strongly to a fixed point of $T$.

Corollary 2.7. Let $X, K,\left\{v_{n}\right\},\left\{u_{n}\right\}$ and $\left\{w_{n}\right\}$ be as in Theorem 2.1 and $T: K \rightarrow K$ is a continuous mapping at a cluster point $p$ of the sequence $\left\{x_{n}\right\}$ defined by (2.1). Let $\left\{a_{n}\right\},\left\{b_{n}\right\},\left\{c_{n}\right\},\left\{a_{n}^{\prime}\right\},\left\{b_{n}^{\prime}\right\},\left\{c_{n}^{\prime}\right\},\left\{a_{n}^{\prime \prime}\right\},\left\{b_{n}^{\prime \prime}\right\}$ and $\left\{c_{n}^{\prime \prime}\right\}$ be the real sequences in $[0,1]$ satisfying

(i) $a_{n}+b_{n}+c_{n}=a_{n}^{\prime}+b_{n}^{\prime}+c_{n}^{\prime}=a_{n}^{\prime \prime}+b_{n}^{\prime \prime}+c_{n}^{\prime \prime}=1$ for all $n \geq 0$;

(ii) $0<\alpha \leq b_{n}<1$ for all $n \geq 0$;

(iii) $\sum_{n \geq 0} b_{n}^{\prime s}<\infty, \sum_{n \geq 0} b_{n}^{\prime \prime s}<\infty, \sum_{n \geq 0} c_{n}<\infty, \sum_{n \geq 0} c_{n}^{\prime s}<\infty$ and $\sum_{n \geq 0} c_{n}^{\prime \prime s}<\infty$, where $s=\min \{1, q-1\}$.

Then the sequence $\left\{x_{n}\right\}$ converges strongly to a fixed point of $T$.

Proof. Let $\delta_{n}=q \lambda^{q-1} a_{n}^{q-1} b_{n}$. Then, for a positive constant $d$, we have $\delta_{n} \geq d>0$ and so, from

$$
q \lambda^{q-1} a_{n}^{q-1} b_{n}\left\|y_{n}-T y_{n}\right\|^{q} \leq\left\|x_{n}-x^{*}\right\|^{q}-\left\|x_{n+1}-x^{*}\right\|^{q}+M_{1} \gamma_{n},
$$

and, consequently,

$$
d \sum_{n \geq 0}\left\|y_{n}-T y_{n}\right\|^{q} \leq\left\|x_{0}-x^{*}\right\|^{q}+M_{1} \sum_{n \geq 0} \gamma_{n}<\infty,
$$

we conclude that

$$
\lim _{n \rightarrow \infty}\left\|y_{n}-T y_{n}\right\|=0
$$

and also

$$
\lim _{n \rightarrow \infty}\left\|x_{n}-T y_{n}\right\|=0
$$


Since $p$ is a cluster point of the sequence $\left\{x_{n}\right\}$, there exists a subsequence $\left\{x_{n_{j}}\right\}$ of $\left\{x_{n}\right\}$ which converges strongly to $p$. Then we have

$$
\begin{aligned}
\left\|y_{n_{j}}-p\right\| & \leq a_{n_{j}}^{\prime}\left\|x_{n_{j}}-p\right\|+b_{n_{j}}^{\prime}\left\|T x_{n_{j}}-p\right\|+c_{n_{j}}^{\prime}\left\|u_{n_{j}}-p\right\| \\
& \rightarrow 0
\end{aligned}
$$

as $j \rightarrow \infty$. Hence we have $y_{n_{j}} \rightarrow p$ as $j \rightarrow \infty$ and so $T y_{n_{j}} \rightarrow T p$ as $j \rightarrow \infty$. Since

$$
\lim _{j \rightarrow \infty}\left\|y_{n_{j}}-T y_{n_{j}}\right\|=\|p-T p\|=0,
$$

it follows that $p \in F(T)$. As in the proof of Theorem 2.1, we obtain

$$
\left\|x_{n+1}-p\right\|^{q} \leq\left\|x_{n}-p\right\|^{q}+M_{1} \gamma_{n} .
$$

Since $M_{1} \sum_{n \geq 0} \gamma_{n}<\infty$, it follows from Lemma 1.7 that $\lim _{n \rightarrow \infty}\left\|x_{n}-p\right\|$ exists. Therefore, since $\lim _{j \rightarrow \infty}\left\|x_{n_{j}}-p\right\|=0$, we have $\lim _{n \rightarrow \infty}\left\|x_{n}-p\right\|=0$. This completes the proof.

Corollary 2.8. Let $X, K,\left\{v_{n}\right\}$ and $\left\{u_{n}\right\}$ be as in Theorem 2.1 and $T: K \rightarrow K$ is a continuous mapping at a cluster point $p$ of the sequence $\left\{x_{n}\right\}$ defined by (2.2). Let $\left\{a_{n}\right\},\left\{b_{n}\right\},\left\{c_{n}\right\},\left\{a_{n}^{\prime}\right\},\left\{b_{n}^{\prime}\right\}$ and $\left\{c_{n}^{\prime}\right\}$ be the real sequences in $[0,1]$ satisfying

(i) $a_{n}+b_{n}+c_{n}=a_{n}^{\prime}+b_{n}^{\prime}+c_{n}^{\prime}=1$ for all $n \geq 0$;

(ii) $0<\alpha \leq b_{n}<1$ for all $n \geq 0$; $1\}$.

(iii) $\sum_{n \geq 0} b_{n}^{\prime s}<\infty, \sum_{n \geq 0} c_{n}<\infty$ and $\sum_{n \geq 0} c_{n}^{\prime s}<\infty$, where $s=\min \{1, q-$ Then the sequence $\left\{x_{n}\right\}$ converges strongly to a fixed point of $T$.

Corollary 2.9. Let $X, K$ and $\left\{v_{n}\right\}$ be as in Theorem 2.1 and $T: K \rightarrow K$ is a continuous mapping at a cluster point $p$ of the sequence $\left\{x_{n}\right\}$ defined by (2.3). Let $\left\{a_{n}\right\},\left\{b_{n}\right\}$ and $\left\{c_{n}\right\}$ be the real sequences in $[0,1]$ satisfying

(i) $a_{n}+b_{n}+c_{n}=1$ for all $n \geq 0$;

(ii) $0<\alpha \leq b_{n}<1$ for all $n \geq 0$;

(iii) $\sum_{n \geq 0} c_{n}<\infty$.

Then the sequence $\left\{x_{n}\right\}$ converges strongly to a fixed point of $T$.

Corollary 2.10. In Corollary 2.7, if a mapping $T: K \rightarrow K$ is a demicontractive mapping which is demicompact at $0 \in K$, then $\left\{x_{n}\right\}$ converges strongly to a fixed point of $T$. 
Proof. Since $\left\{y_{n}\right\}$ is bounded and the sequence $\left\{y_{n}-T y_{n}\right\}$ converges strongly to 0 , then, by the demicompactness of $T$, there exists a subsequence $\left\{y_{n_{j}}\right\}$ of $\left\{y_{n}\right\}$ which converges strongly to a point $p \in F(T)$. From

$$
\begin{aligned}
\left\|x_{n_{j}}-p\right\| & \leq\left\|x_{n_{j}}-y_{n_{j}}\right\|+\left\|y_{n_{j}}-p\right\| \\
& \leq\left\|y_{n_{j}}-p\right\|+b_{n_{j}}^{\prime}\left\|x_{n_{j}}-T x_{n_{j}}\right\|+c_{n_{j}}^{\prime}\left\|x_{n_{j}}-u_{n_{j}}\right\| \\
& \leq\left\|y_{n_{j}}-p\right\|+M_{*}\left(b_{n_{j}}^{\prime}+c_{n_{j}}^{\prime}\right) \\
& \rightarrow 0
\end{aligned}
$$

as $j \rightarrow \infty$, it follows that $\left\{x_{n_{j}}\right\}$ converges strongly to $p$. The rest now follows as in the proof of Corollary 2.7. This completes the proof.

Corollary 2.11. In Corollary 2.8, if $T: K \rightarrow K$ is a demicontractive mapping which is demicompact at $0 \in K$, then the sequence $\left\{x_{n}\right\}$ converges strongly to a fixed point of $T$.

Corollary 2.12. In Corollary 2.9, if $T: K \rightarrow K$ is a demicontractive mapping which is demicompact at $0 \in K$, then the sequence $\left\{x_{n}\right\}$ converges strongly to a fixed point of $T$.

Remark 2.13. ([16]) (1) By setting $c_{n}=c_{n}^{\prime}=c_{n}^{\prime \prime}=0$ in our results, we can show that the usual Mann and Ishikawa iteration methods converge (strongly and weakly, according to the conditions) to a fixed point of $T$.

(2) Our results do not depend on Opial's condition.

(3) Our results hold, in particular, in the Lebesgue $L_{p}(p \neq 2)$ spaces.

(4) Suppose that $A: X \rightarrow X$ is a continuous linear operator, with zero as an eigenvalue, satisfying the condition that, for all $z \in D(A)$ and a positive constant $\lambda$,

$$
\langle A z, j(z)\rangle \geq \lambda\|A z\|^{2} .
$$

Suppose, further, that $f \in R(A)$. Then we may apply our results to prove that the three-step, Ishikawa and Mann iteration methods with errors converge strongly to a solution of the equation $A x=f$.

Example 2.14. ([2]) Let $T:[0,3] \rightarrow[0,3]$ be defined by

$$
T x= \begin{cases}2 x-4 & \text { if } x \in[2,3], \\ 0 & \text { if } x \in[0,2] .\end{cases}
$$

Then $T$ is demicontractive and Lipschitzian but it is not even a pseudocontractive mapping. 


\section{References}

[1] R.P. Agarwal, Y.J. Cho, J. Li, N.J. Huang, Stability of iterative procedures with errors approximating common fixed points for a couple of quasicontractive mappings in $q$-uniformly smooth Banach spaces, J. Math. Anal. Appl., 272 (2002), 435-447.

[2] D. Boonchari, S. Saejung, Construction of common fixed points of a countable family of $k$-demicontractive mappings in arbitrary Banach spaces, Appl. Math. Comput., 216 (2010), 173-178.

[3] F.E. Browder, W.V. Petryshyn, Construction of fixed points of nonlinear mappings in Hilbert space, J. Math. Anal. Appl., 20 (1967), 197-228.

[4] C.E. Chidume, The solution by iteration of nonlinear equations in certain Banach spaces, J. Nigerian Math. Soc., 3 (1984), 57-62.

[5] C.E. Chidume, An iterative method for nonlinear demiclosed monotonetype operators, Dynamical Systems Appl., 3 (1994), 349-356.

[6] J.B. Diaz, F.T. Metcalf, On the set of subsequential limit points of successive approximations, Trans. Amer. Math. Soc., 135 (1969), 459-485.

[7] K. Goëbel, W.A. Kirk, T.N. Shimi, A fixed point theorem in uniformly convex spaces, Boll. Un. Mat. Ital, 7 (1973), 67-75.

[8] T.L. Hicks, J.D. Kubicek, On the Mann iteration process in Hilbert spaces, J. Math. Anal. Appl., 59 (1977), 498-504.

[9] S. Ishikawa, Fixed point by a new iteration method, Proc. Amer. Math. Soc., 44 (1974), 147-150.

[10] R. Kannan, Fixed point theorems in reflexive Banach spaces, Proc. Amer. Math. Soc., 38 (1974), 111-118.

[11] L.S. Liu, Ishikawa and Mann iteration process with errors for nonlinear strongly accretive mappings in Banach spaces, J. Math. Anal. Appl., 194 (1995), 114-125.

[12] Q.H. Liu, The convergence theorems of the sequence of Ishikawa iterates for hemicontractive mappings, J. Math. Anal. Appl., 148 (1990), 55-62.

[13] Q.H. Liu, Convergence theorems of the sequence of iterates for asymptotically demicontractive and hemicontractive mappings, Nonlinear Anal., 26 (1996), 1835-1842. 
[14] W.R. Mann, Mean value methods in iteration, Proc. Amer. Math. Soc., 4 (1953), 506-510.

[15] Şt. Măruşter, The solution by iteration of nonlinear equations in Hilbert spaces, Proc. Amer. Math. Soc., 63 (1977), 69-73.

[16] C. Moore, Iterative approximation of fixed points of demicontractive maps, preprint, http, //www.ictp.trieste.it/ ${ }^{\sim}$ pub.

[17] S.A. Naimpally, K.L. Singh, Extensions of some fixed point theorems of Rhoades, J. Math. Anal. Appl., 96 (1983), 437-446.

[18] M.O. Osilike, Iterative method for nonlinear monotone-type operators in uniformly smooth Banach spaces, J. Nigerian Math. Soc., 12 (1993), 73-79.

[19] W.V. Petryshyn, T.E. Williamson Jr., Strong and weak convergence of the sequence of successive approximations for nonexpansive mappings, $J$. Math. Anal. Appl., 43 (1973), 459-497.

[20] B.E. Rhoades, Comments on two fixed point iteration methods, J. Math. Anal. Appl., 56 (1976), 741-750.

[21] H.F. Senter, W.G. Dotson Jr., Approximating fixed points of nonexpansive mappings, Proc. Amer. Math. Soc., 44 (1974), 375-380.

[22] K.K. Tan, H.K. Xu, Approximating fixed points of nonexpansive mappings by the Ishikawa iteration process, J. Math. Anal. Appl., 178 (1993), 301308.

[23] X.L. Weng, The iterative solution of nonlinear equations in certain Banach spaces, J. Nigerian Math. Soc., 11 (1992), 1-7.

[24] C.S. Wong, Fixed points and characterization of certain maps, Pacific J. Math., 54 (1974), 305-312.

[25] H.K. Xu, Inequalities in Banach spaces with applications, Nonlinear Anal., 16 (1991), 1127-1138.

[26] Y. Xu, Ishikawa and Mann iterative processes with errors for nonlinear strongly accretive operator equations, J. Math. Anal. Appl. 224 (1998), 91-101. 
[27] Z.B. Xu, G.F. Roach, Characteristic inequalities of uniformly convex and uniformly smooth Banach spaces, J. Math. Anal. Appl., 157 (1991), 189210. 